\title{
Uptake and distribution of carboxylated quantum dots in human mesenchymal stem cells: cell growing density matters
}

Gabrielis Kundrotas ${ }^{1,4}$ (D) Vitalijus Karabanovas ${ }^{2,3}$ (D) Marijus Pleckaitis ${ }^{2}$, Marina Juraleviciute², Simona Steponkiene ${ }^{2}$, Zivile Gudleviciene ${ }^{1}$ and Ricardas Rotomskis ${ }^{2,5^{*}}$

\begin{abstract}
Background: Human mesenchymal stem cells (MSCs) have drawn much attention in the field of regenerative medicine for their immunomodulatory and anti-inflammatory effects. MSCs possess specific tumor-oriented migration and incorporation highlighting the potential for MSCs to be used as an ideal carrier for anticancer agents. Bone marrow is the main source of MSCs for clinical applications. MSCs tracking in vivo is a critical component of the safety and efficacy evaluation of therapeutic cell products; therefore, cells must be labeled with contrast agents to enable visualization of the MSCs migration in vivo. Due to their unique properties, quantum dots (QDs) are emerging as optimal tools in long-term MSC optical imaging applications. The aim of this study was to investigate the uptake dynamics, cytotoxity, subcellular and extracellular distribution of non-targeted carboxylated quantum dots in human bone marrow MSCs at different cell growing densities.
\end{abstract}

Results: QDs had no negative impact on MSC viability throughout the experiment and accumulated in all observed cells efficiently; however, in some MSCs QDs induced formation of lipid droplets. At low cell growing densities QDs distribute within MSCs cytoplasm already after $1 \mathrm{~h}$ of incubation reaching saturation after $6 \mathrm{~h}$. After $24 \mathrm{~h}$ QDs localize mainly in the perinuclear region of the cells in endosomes. Interestingly, in more confluent culture QDs localize mostly outside MSCs. QDs abundantly mark MSC long filopodia-like structures attaching neighboring cells. At high cell density cultivation, we for the first time demonstrated that carboxylated QDs localize in human bone marrow MSC extracellular matrix. Moreover, we observed that average photoluminescence lifetime of QDs distributed in extracellular matrix are longer than lifetimes of QDs entrapped in endocytic vesicles; thus, for the first time showing the possibility to identify and distinguish localization of QDs in various extracellular and intracellular structures using fluorescence-lifetime imaging microscopy without additional staining assays.

Conclusion: Carboxylated QDs can be used as nonspecific and effective dye for staining of human bone marrow MSCs and their specific extracellular structures. These results are promising in fundamental stem cell biology as well as in cellular therapy, anticancer drug delivery and tissue engineering.

Keywords: Mesenchymal stem cells, Filopodia-like structures, Extracellular matrix, Labeling, Quantum dots, Fluorescence imaging, Fluorescence-lifetime imaging microscopy

\footnotetext{
*Correspondence: ricardas.rotomskis@nvi.lt

2 Biomedical Physics Laboratory, National Cancer Institute, Baublio Str. 3b, 08406 Vilnius, Lithuania

Full list of author information is available at the end of the article
} 


\section{Background}

Human mesenchymal stem cells (MSCs) are a diverse subset of fibroblast-like precursor cells with high selfrenewal capacity present in the stromal fraction of many adult tissues [1]. During the last decade MSCs have drawn much attention from basic and translational investigators in the field of regenerative medicine, mainly due to their multipotent differentiation capacity, immunomodulatory and anti-inflammatory effects [2]. Though the nature and functions of MSCs remain not fully clear, several clinical trials have underscored their effectiveness in treating different illnesses, including hematological, inflammatory, cardiovascular, bone and cartilage, neurological and autoimmune diseases [3].

MSCs are particularly promising in cancer therapy. The specific tumor and their metastases-oriented migration and incorporation of MSCs have been demonstrated in various pre-clinical models, highlighting the possibility of modifying these cells to express anticancer molecules and using them as an ideal carrier for anticancer agent delivery [4]. The role of MSC as carriers of drug delivery systems offers an alternative therapeutic approach capable of overcoming clinical restrictions related to the systemic administration of antitumor agents including cytokines, interferons or pro-drugs with short half-life and high toxicity [5]. Recently, the use of modified MSCs as therapy vehicles for the treatment of solid tumors has progressed to the first generation of clinical trials [6].

Cell tracking is a critical component of the safety, efficacy and mechanism of action evaluation of therapeutic cell products [7]. To track MSCs, cells must be labeled with a contrast agent prior to transplantation to make them visible within the body [8]. Therefore, fluorescent imaging [9] and magnetic resonance imaging (MRI) [10] technologies based on nanoparticles have been developed to monitor MSC after injection. Nanotechnology-based cell-tracking methods provide non-toxic, non-invasive, clinically applicable solutions for long-term monitoring of cells post-injection [11]. Superparamagnetic iron oxide (SPIO) nanoparticle based MRI is among the most widely employed for in vivo monitoring of the stem cells $[12,13]$. However, limited image resolution makes it challenging to accurately detect small numbers of cells after transplantation with MRI [14]. Also, MRI may overestimate the true size of the MSC grafts [15]. Moreover, SPIO-based MRI may not be suitable for longterm tracking of transplanted MSCs in vivo compared to MSCs labeled with fluorescent protein [16].

The most popular labelling markers for fluorescence imaging are organic dyes. However, most organic dyes can only be used for short-term imaging because of photobleaching effect [17]. Another emerging type of fluorescent labels are quantum dots (QDs) which are fluorescent semiconductor nanoparticles with unique optical and chemical features which make them useful as fluorescent tags for long-term in vitro and in vivo cell imaging applications. QDs have narrow band emission spectra and broad excitation spectra and are resistant to chemical and metabolic degradation [18]. QDs possess improved signal brightness as well as enhanced resistance to photobleaching compared to conventional organic and protein fluorophores [19].

Using QDs for the labeling and tracking purposes of human mesenchymal stem cells have been firstly demonstrated in the study of Hsieh et al. [20]. Furthermore, QDs were specifically modified with biologically active molecules making the imaging of particular MSCs structures possible [21]. Another potential application of bioconjugated QDs is effective regulation of MSCs differentiation [22], which is important for understanding of MSCs behaviors in vitro and in vivo [23]. Recently, we have shown that non-targeted carboxyl-coated QDs are biocompatible with human skin MSCs, not affecting cell viability, proliferation, immunophenotype and ability to differentiate [24]. Moreover, in 3D spheroid co-culture and human tumor xenograft model we have demonstrated that MSCs nanoengineered with QDs could serve as a vehicle for targeted drug delivery to metastatic cancer $[25,26]$. These results are in compliance with observations of other groups. Ohyabu et al. showed that QDs are efficient, genetically noninvasive, nontoxic, and functionally inert way to label human MSCs [27]. Tao et al. demonstrated that human bone marrow MSCs labeled with QDs remain viable on biological sutures transplanted in vivo [28]. Based on these observations, we hypothesize that carboxylated QDs would be an optimal tool for human MSC labeling and imaging. Bone marrow derived MSCs are the most frequently investigated cell type and often designated as the gold standard [29]. However, significant number of MSCs is needed for effective cell mediated therapy [30], thus it is necessary to label large amount of MSCs with QDs simultaneously.

In this study, we for the first time investigated the distribution of non-targeted carboxylated quantum dots with emission peak at $625 \mathrm{~nm}$ in human bone marrow MSC culture at different cell growing density. Surprisingly, at 20,000 cells $/ \mathrm{cm}^{2}$ cell growing density, these nanoparticles localized not only inside the MSCs, but also on cell protrusions, as well as irregularly outside the cells highlighting extracellular matrix.

\section{Results}

QDs intracellular uptake dynamics and biological effects on MSCs

After isolation from bone marrow, MSCs for characterization were expanded in vitro until passage 3. Before QDs 
labeling experiments, identity of MSCs were confirmed by adherence to plastic, morphology, immunophenotype, proliferation capacity and genomic stability. Results of MSC analysis we have published previously [31], showing long spindle-shaped or flat fibroblast like MSC morphology with 99\% of the cells stained positive for surface markers CD44, CD73, CD90 and CD105 and more than $98 \%$ of the cells stained negative for antigens CD11b, CD19, CD45, CD34 and HLA-DR. Viability of cultivated and non-treated MSCs varied between 87.0 and $96.6 \%$ in this study (data not shown). To evaluate the temporal accumulation dynamics of QDs into MSCs at 5000 cells/ $\mathrm{cm}^{2}$ seeding density, the photoluminescence (PL) intensity of QDs at $625 \mathrm{~nm}$ was registered by the means of flow cytometry until $24 \mathrm{~h}$ of incubation. The intracellular QDs photoluminescence intensity signal gradually increased and started to saturate after $6 \mathrm{~h}$ of incubation. Saturation remained throughout the rest time of the experiment (Fig. 1a).

The time dependent cytotoxicity at QDs concentration of $8 \mathrm{nM}$ was assessed. Incubation of MSCs with QDs did not change cell viability significantly during the accumulation experiments (accumulation time 1-24 h) and varied within $8 \%$ error limits (Fig. 1b). It should be stressed that labeling with QDs did not change MSCs morphology and no obvious features of apoptosis (cell rounding, cell and nuclei fragmentation) were observed (Fig. 1c).

\section{QDs localization in MSC culture}

Accumulation of QDs in MSC after 1, 3, 6 and $24 \mathrm{~h}$ of incubation was investigated. Three different types of images of accumulation of QDs [inside MSCs (Fig. 2), on MSC particular surface parts (Fig. 3) and outside the MSCs (Fig. 5)] dependent on seeded cells density were detected

Vesicle-type structures (endosomes) filled with QDs and spread throughout the cytoplasm already $1 \mathrm{~h}$ after introduction were detected (Fig. 2a). The number and size of these vesicles inside the cells increases with incubation time together with the increasing of the intensity of PL of QDs (Fig. 2). After $24 \mathrm{~h}$ treatment in some cells we observed large vesicles positive for Oil Red O staining (Fig. 2g-i) without nanoparticles, clustered around the

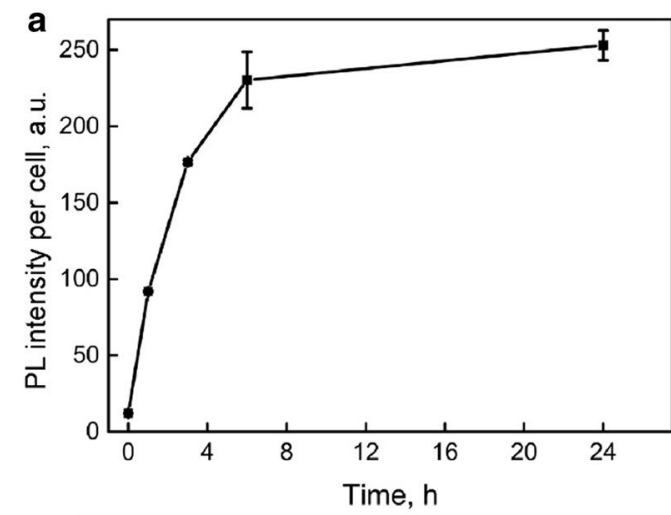

C
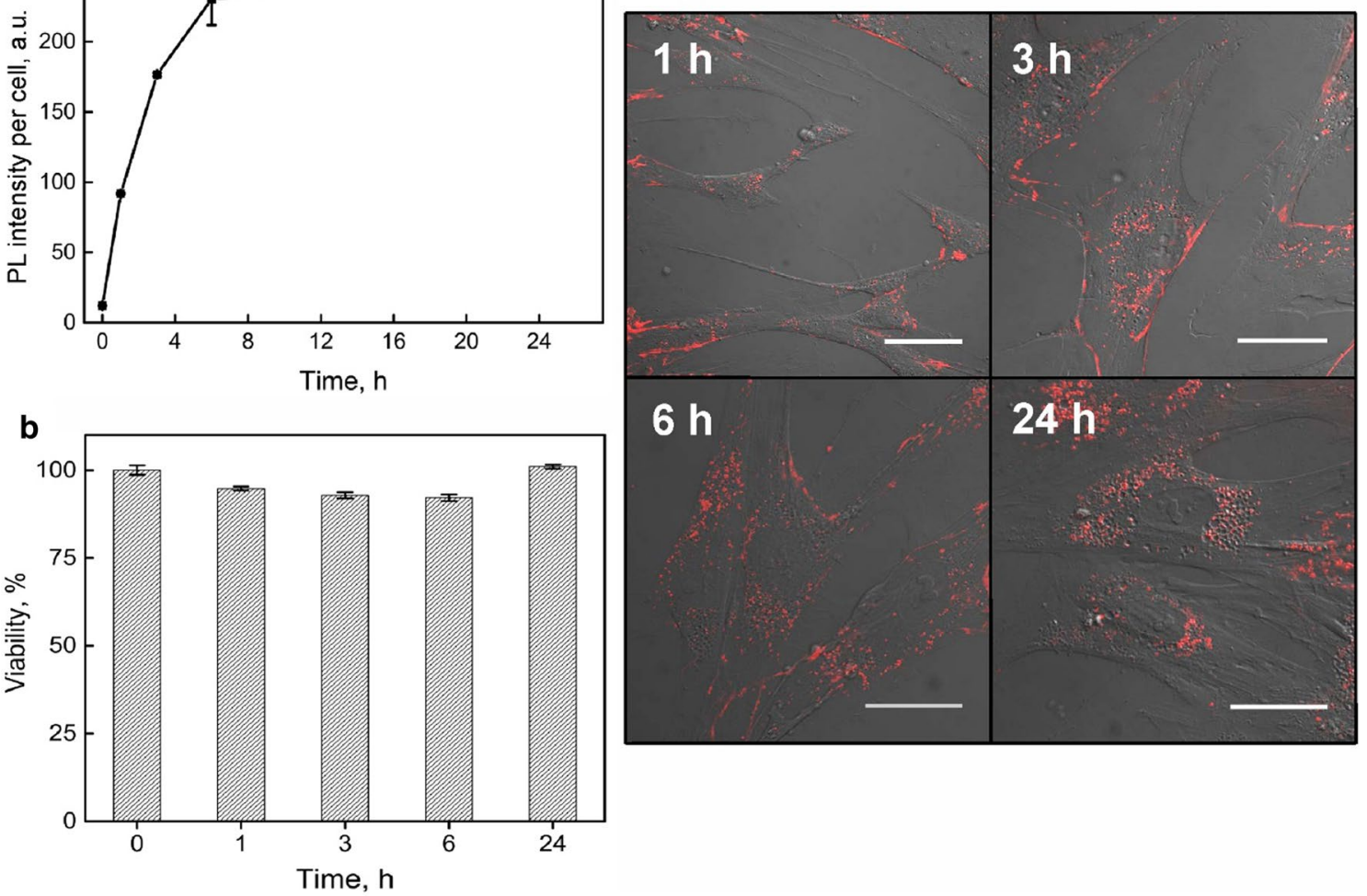

Fig. 1 Carboxylated quantum dots intracellular uptake dynamics and biological effects on MSCs. a Dynamics of accumulation for QDs in MSCs. b MSCs viability at different incubation times with QDs. c MSCs morphology and intracellular distribution of QDs after various times of incubation. Red color exhibits QDs distribution in MSCs culture 


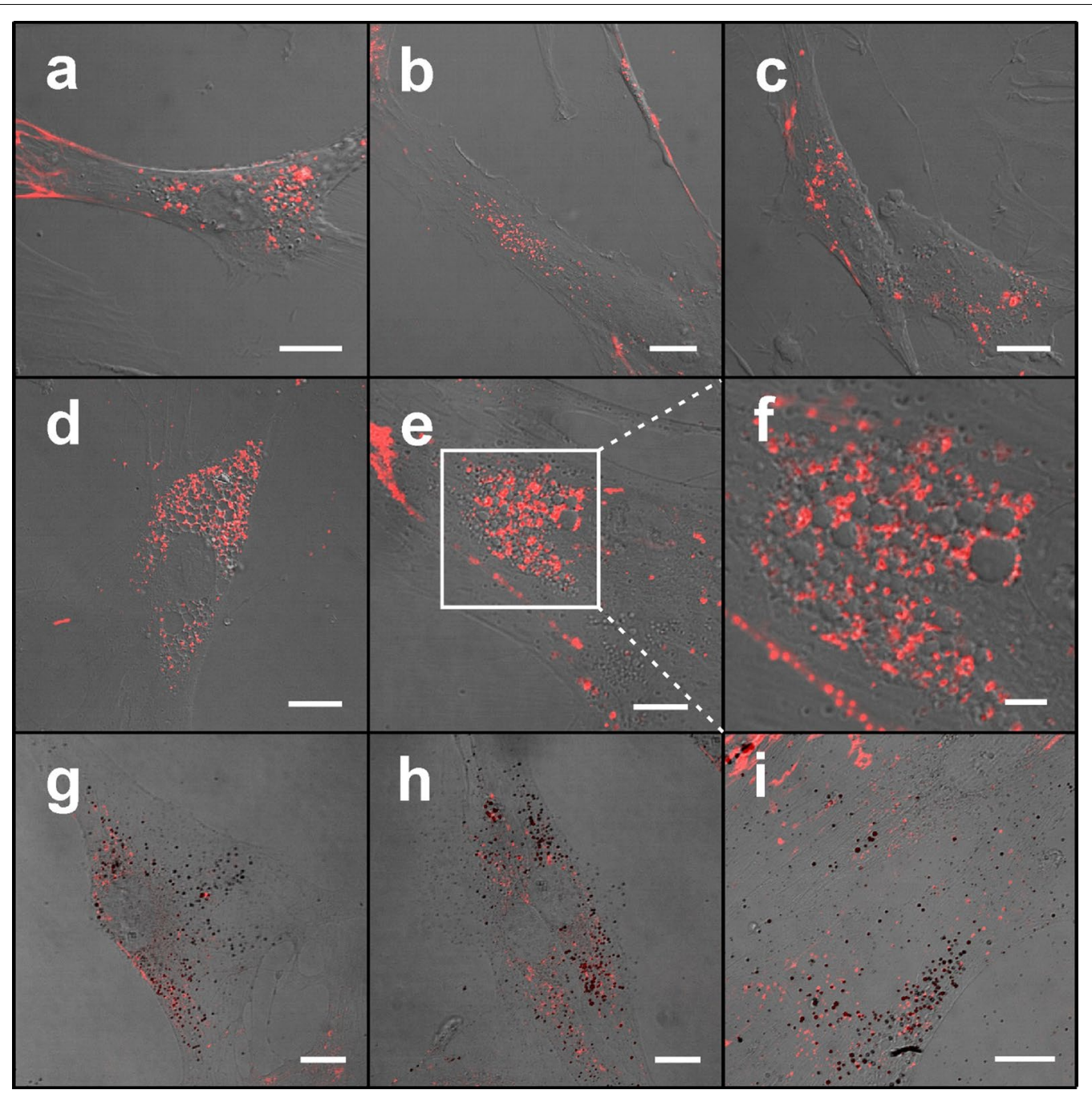

Fig. 2 Distribution of carboxylated quantum dots in MSCs at different incubation times. Confocal fluorescence microscopy images showing the distribution of QDs in MSCs after $1 \mathrm{~h}(\mathbf{a}), 3 \mathrm{~h}(\mathbf{b}), 6 \mathrm{~h}(\mathbf{c}), 24 \mathrm{~h}(\mathbf{d}-\mathbf{f})$ incubation. Enlarged part of MSC containing hollow vesicles surrounded by smaller QDs-fulfilled vesicle-type structures after $24 \mathrm{~h}$ incubation (f) shown with white rectangle in e. MSCs incubated with QDs and additionally stained with Oil Red O dye show adipogenic-like phenotype of MSCs $24 \mathrm{~h}$ after treatment with QDs $(\mathbf{g}-\mathbf{i})$. Scale bars $15 \mu \mathrm{m}(\mathbf{a}-\mathbf{e}, \mathbf{g}-\mathbf{i})$ and $5 \mu \mathrm{m}(\mathbf{f})$

nucleus and surrounded with endosomes filled with QDs (Fig. 2e, f).

Confocal fluorescence microscopy analysis showed that in more confluent MSC culture seeded at 20,000 cells/ $\mathrm{cm}^{2}$ QDs localize not only inside the cells, but attach mainly on some surface structures of the main cell body and on the long filopodia-like structures which contact neighboring cells, as well as on the complex extracellular structures which are not characteristic for single MSCs (Fig. 3a-d) and which are oriented not in the same directions as the cells themselves (Fig. $3 \mathrm{e}-\mathrm{h}$ ). Also see Additional files 1 and 2.

After cell harvesting from flask surface, we detected that QDs remain attached to extracellular structures and the use of trypsin does not affect their properties (data not shown). The differential interference contrast (DIC) microscopy was applied to observe these structures, because extracellular structures of non-labeled (with QDs) human MSCs were not detectable with conventional bright-field microcopy.

After $24 \mathrm{~h}$ incubation, confocal immunofluorescence microscopy images demonstrated that quantum dots extracellularly co-lozalize with the CD44 and fibronectin, and intracellularly with the transferrin (Fig. 4b-d).

Confocal laser scanning microscopy analysis identified QDs on the middle and the upper scanning layers of the picture in $\mathrm{Z}$ dimension localizing above MSC intercellular actin and nucleus (Fig. 5, Additional files 3 and 4). 

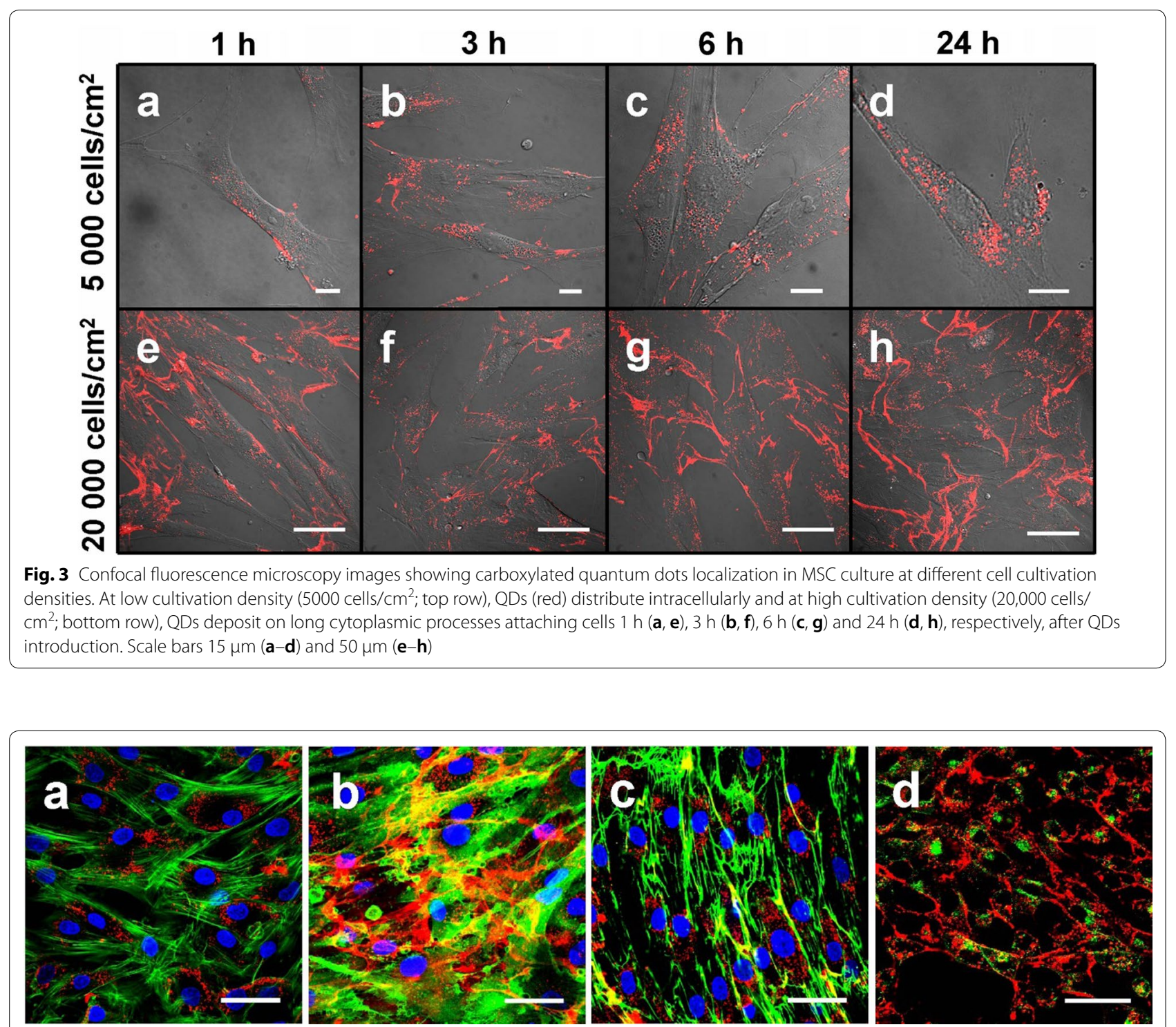

Fig. 4 Staining of MSC intracellular and extracellular molecules. MSCs labelled with actin staining Alexa-Fluor $488 \mathrm{~nm}$-conjugated phalloidin (green, a), mouse anti-human CD44 antibody conjugated with Alexa-Fluor 488 (green, b), Gibco fibronectin (green, c) and Alexa-Fluor 488 nm-conjugated transferrin (green, d) after $24 \mathrm{~h}$ of incubation with QDs (red). Scale bars for all images $50 \mu \mathrm{m}$

Fluorescence lifetime imaging microscopy (FLIM) images were registered at four defined time gates that allowed us to study the spatial localization of QDs (Fig. 6). The presence of time-related differences in the mean PL lifetime intracellular distribution implies that the intravesicular QDs are surrounded with different microenvironments [32, 33]. In contrast, if the QDs surrounding media were homogenous, the FLIM images would be identical and only the PL intensity would differ. Two regions of interest (ROI) were inspected from each FLIM image at various incubation times which enabled the identification of how the different phases in uptake of QDs affects PL of these nanoparticles. The results showed that PL lifetimes of QDs accumulated in extracellular matrix or membrane were longer than lifetimes of QDs in endocytic vesicles. PL decayed even more during maturation of endolysosomal structures [33]. In addition, the results revealed the tendency for PL lifetimes to become shorter the longer time of incubation with QDs is which supports the idea of spatial heterogeneity of intracellular vesicles filled with carboxyl-coated QDs. Mean PL lifetimes of QDs distributed on the extracellular matrix were from $\sim 18.5 \mathrm{~ns}$ after $1 \mathrm{~h}$ of incubation decaying to $\sim 17.1 \mathrm{~ns}$ after $24 \mathrm{~h}$. For QDs accumulated on membrane of MSCs mean PL 


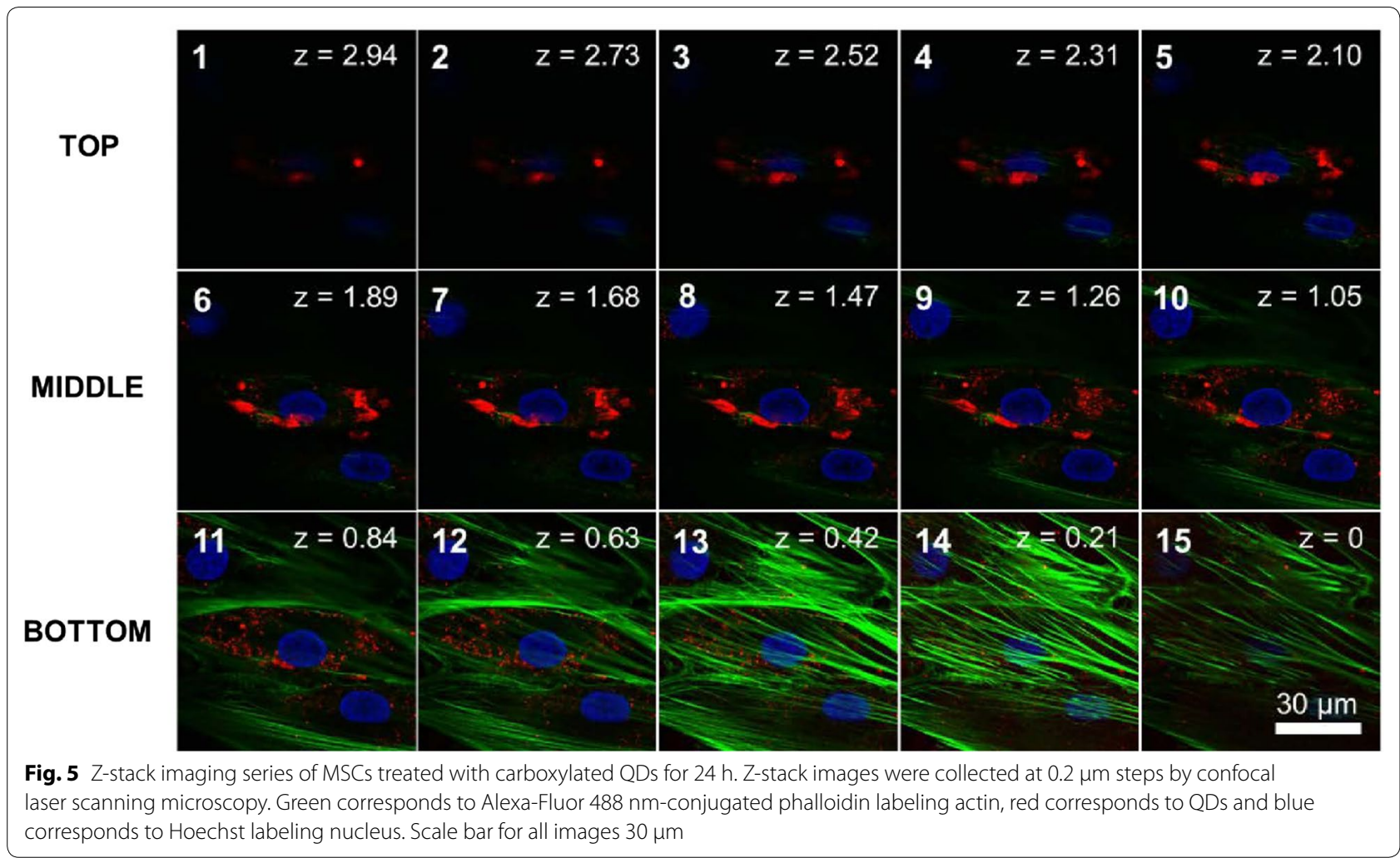

lifetime $1 \mathrm{~h}$ after introduction of QDs also was $\sim 18.5 \mathrm{~ns}$. However, it decayed less during time and after $24 \mathrm{~h}$ mean lifetime of QDs was about 17.8 ns. Finally, FLIM allowed distinguishing of QDs accumulated intracellularly. PL decayed from $\sim 17.5$ to $\sim 14.3 \mathrm{~ns}$ after $1 \mathrm{~h}$ and $24 \mathrm{~h}$ of incubation with QDs, respectively.

\section{Discussion}

Previously we have shown that human bone marrow MSCs isolated from patients by a novel donor-friendly methodology [34] retain their genomic stability and stemness characteristics in early culture passages [31]. We confirmed MSCs identification and homogeneity by adhesion, morphological and flow cytometry [31] criteria as recommended by the International Society for Cellular Therapy [35]. Real-time in vivo cell tracking can be performed by labeling cells with probes that enter the cell by active or passive transport and are trapped intracellularly [36]. Despite the increasing popularity of QDs as cell labeling agents, their potential cytotoxicity remains a major issue among academic, industrial, and regulatory communities [37].

As it is seen from our experiments, the human bone marrow stem cells accumulate the QDs and do not exhibit toxicity after $24 \mathrm{~h}$ of incubation at concentration $8 \mathrm{nM}$. We did not detect any increase of debris or dead cells (Fig. 1c) showing no cytotoxic effect of QDs on the cells. MSC viability was considerably higher than $80 \%$ throughout the experiment (Fig. 1b); therefore, QDs can be recognized as biocompatible [38]. We consider the experiment time of $24 \mathrm{~h}$ to be sufficient because MSCs are expected to be transplanted within $24 \mathrm{~h}$ of QD labeling [39]. To sum up, here we show that human MSCs are efficiently labeled with QDs with no cytotoxity.

We also showed that human bone marrow MSCs uptake QDs efficiently. Cellular uptake of QDs increases during the first hours of incubation and reaches plateau within $6 \mathrm{~h}$ (Fig. 1a). These results are consistent with QDs uptake dynamics in human skin MSCs [26]. Several reasons could lead to the saturation. MSCs could stop up-taking more QDs due to internal biochemical mechanisms (for example, due to no free membrane endocytosis receptors) or because of exhaustion of QDs in the medium.

Moreover, specific accumulation of QDs were detected in stem cell in comparison with other cell type cultures [40]. The extraordinary extracellular and intracellular distribution of QDs were detected (Fig. 3) whereas in other cell type cultures QDs distribute in vesicles and distributed uniformly in the cell under prolonged incubation [40]. Accumulated in MSCs culture QDs localize at three different levels (inside MSCs, on particular MSC surface 


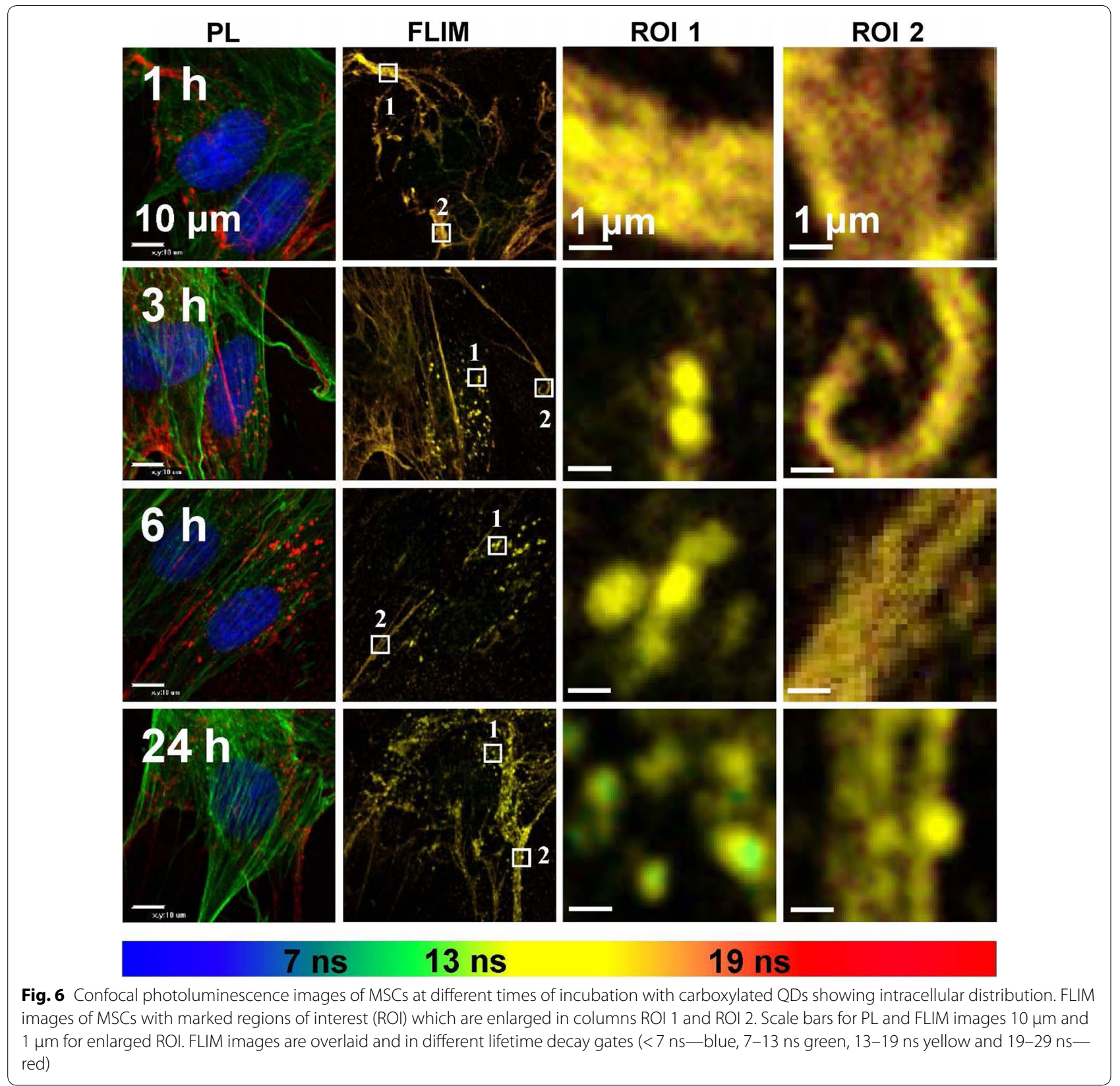

parts and outside the MSCs) dependent on seeded cells density. At each level QDs localize irregularly and thus highlight special structures of in vitro cultivated human bone marrow MSCs. In addition, here we confirmed high expression of CD44 (Fig. 4b) which is specific marker of stemness and mesenchymal lineage [41].

QDs label MSCs specifically because we did not determine QDs in the background throughout the experiment. Practically, all MSCs displayed red fluorescence signals showing that all the cells were labeled with QDs. Intracellular QDs are already detectable at $1 \mathrm{~h}$ of incubation. The brightness of signals increased until $6 \mathrm{~h}$ and remained such until $24 \mathrm{~h}$ (Fig. 1c). These fluorescence confocal microscopy results are in line with flow cytometry results (Fig. 1a). We noticed that QDs accumulate in human bone marrow MSCs spontaneously similar like in mouse fibroblasts which uptake QDs by natural endocytosis [32] and distribute throughout the cytosol (Fig. 2). However, we observed the differences already at the first time point of observation. Vesicles filled with QDs distribute within MSCs already $1 \mathrm{~h}$ after introduction and no QDs were detected on plasma membrane (Fig. 2a). Our results 
correlate with results of other teams showing that QDs with emissions at $520 \mathrm{~nm}, 525 \mathrm{~nm}, 605 \mathrm{~nm}$ and $645 \mathrm{~nm}$ firstly disperse throughout the cytoplasm, then after $24 \mathrm{~h}$ localize mainly in the perinuclear area of bone marrow MSCs in endosome-like structures [20, 42, 43]. Intracellular co-localization of QDs with transferrin after $24 \mathrm{~h}$ shows that quantum dots accumulate in MSC by clathrin-dependent endocytosis pathway [32] and afterwards fuse with transferrin containing endosomes (Fig. 4d). The presence of endosomes on actin filaments demonstrates the transportation of these vesicles, but also implies that once inside the endosomes, QDs cannot escape and stain intracellular structures (Fig. 4a). Cumulatively, these results demonstrate that human MSCs are efficiently labeled with QDs and suggest $6 \mathrm{~h}$ of MSCs incubation with QDs to be optimal.

Moreover, we found that in some vesicles clustered around the nucleus QDs do not accumulate and these were surrounded with smaller vesicles filled with QDs. It is possible that these two types of vesicles do not fuse because we haven't detected their fusion throughout the experiment. After staining with Oil Red O some of these QD-negative vesicles were seen as bright red spheres, a characteristic of human MSC adipocytic phenotype [44]. Lipid droplets consist of apolar lipids and other hydrophobic substances and are surrounded by amphiphilic proteins [45]. QDs have attached hydrophilic carboxyl groups and that could explain why these vesicles do not fuse. However, in our study MSCs did not change in morphology from the fibroblastic shape into and adipocytelike spherical shape what is typical for adipocytes derived from human MSCs [46]. Lipid droplets were more prominent in $24 \mathrm{~h}$ treated MSCs (Fig. 2g-i), leading to conclusion that these structures are QDs induced. Saulite et al. [24] showed that carboxyl-coated QD655 do not induce human skin MSC spontaneous differentiation and do not alter ability to differentiate into adipocytes. Meanwhile, it was demonstrated that silver nanoparticles enhance human bone marrow MSC adipogenesis [47] and graphene QDs enhance adipogenic differentiation of rat bone marrow MSCs [48]. We presume that adipogenic features observed in this study may reflect some degree of the intrinsic bone marrow MSC adipocytic commitment [49] that might have been promoted by QDs.

Our next series of experiments were focused on QDs localization on MSC surface and extracellular structures that are obviously seen in fluorescence imaging at 20,000 cells $/ \mathrm{cm}^{2}$ MSC culture density (Fig. 3e-h). The localization of the QDs on the surface of subconfluencial MSCs was exclusive. QDs co-localized with cell surface marker CD44 (Fig. 4b), confirming QDs position on MSC outer membrane. It should be noted that QDs concentrated mainly on the long cell membrane protrusions of connected adherent cells (Fig. 3e-h). Such layout of QDs is not characteristic for other cell types, such as fibroblasts or cancer cell lines [40], indicating specificity of MSC projections. Sectioning with a confocal laser scanning microscope showed that QDs localize on the middle and upper scanning layers lacking MSC cellular actin, thus indicating the extracellular position (Fig. 5). It was confirmed by revealing QDs colocalization with fibronectin (Fig. 4c) which is essential and ubiquitous extracellular matrix (ECM) glycoprotein [50]. These observations were further confirmed by showing QDs localization with CD44 (Fig. 4b) which has been described as the main receptor that modulates cell-ECM interactions [51]. Chen et al. demonstrated that CD44 molecules on human umbilical cord MSCs are predominantly located on the peak of the membrane protrusions, which may enhance CD44 binding properties to the ECM [52]. CD44 mediate cell adhesion to and migration through the ECM, and Ke et al. showed that CD44 is involved in migration of human umbilical cord MSCs [53]. Cumulatively, these results strongly point out that these complex structures are likely to be the ECM. In this study, for the first time to our knowledge we demonstrate the MSC-deposited ECM by labeling it with non-targeted carboxylated quantum dots. QDs are negatively charged; therefore, it is likely that QDs interact nonspecifically with positive sites of ECM, thus revealing its full picture.

ECM is an important component of the cellular microenvironment, supplying critical biochemical and physical signals to initiate and sustain cellular functions [54]. It is known that MSCs in vitro alter their niche to allow cell attachment by synthesizing their own ECM proteins. Several studies have shown that human bone marrow MSCs deposit ECM in vitro [55, 56]. It might at least partially explain the QD-negative vesicles we detected in the perinuclear region (Fig. 2d) which are associated with intense protein synthesis [57]. We noticed that ECM is detectable only in cell culture with higher confluence (Fig. 3e-h). It is in compliance with literature data showing that confluent human MSCs preferentially express genes for ECM proteins when comparing with pre-confluent culture [58]. We also noted that the denser ECM is, the lesser QDs are inside the cells (Fig. 3). Transferrin still enters MSCs and partially co-localizes with QDs (Fig. 4d); therefore, ECM is not likely to act as a barrier for QDs to get to the cell membrane. All these findings indicate that QDs could be secreted together with ECM proteins.

FLIM gives an efficient and unique approach to study cells and their interactions with nanoparticles because of its versatility, specificity and relatively high sensitivity. By analyzing the characteristics of PL, it becomes possible to visualize and study complex dynamic events 
and interactions of nanoparticles with surrounding environment in cells, organelles and sub-organelle components within the biological specimen [59, 60]. PL spectra of QDs, localized inside or outside the cells, usually can be similar or hardly distinguishable. Thus, FLIM allows making a distinction between different phases of incubation and accumulation of QDs. In previous our study, we showed that QDs accumulation is not only time-dependent but also depends on localization inside/outside the cells. Moreover, the changes in mean PL lifetime of QDs registered during their accumulation most likely can be addressed to the variations in biomolecular composition and acidity of the microenvironment altering the molecular interactions with QDs, which can affect PL properties of QDs [33]. These findings support the results of our current study, in which FLIM analysis results show that QDs located outside MSCs have longer lifetimes than QDs located inside the cells (Fig. 6). We denoted the advantages of applying FLIM for effectively studying time-dependent accumulation and dynamics of QDs within MSCs both intracellularly and extracellularly. Even more, MSCs labeling technology with QDs can be possibly used for ECM identification without additional immunofluorescent staining of MSCs.

Interestingly, ECM is located not in the same direction as underlying MSCs and the components in ECM do not show any specific orientation (Fig. 3e-h). These results conform with recently published study which demonstrated the increase of bovine bone marrow MSC ECM production, but the lack of fiber organization capabilities when comparing to meniscal fibrochondrocytes. The researchers showed that co-culture can be used as a technique of balancing the synthetic properties of MSCs and the matrix remodeling capabilities of other cell types for tissue engineering applications [61]. It is known that MSCs can remodel their environment by simultaneous degradation of the scaffolds and deposition of newly synthesized ECM [62]. Our study shows that QDs have a huge potential in tissue engineering as nonspecific dye for staining dynamic ECM of viable human bone marrow MSCs.

To our knowledge, Auletta et al. were the first to label human bone marrow MSCs with Qtracker 625 QDs. The researchers investigated the biodistribution and mechanism of action of these cells on graft-versus-host disease and graft-versus-leukemia activity following bone marrow transplantation in mice model. By using novel microscopic cryo-imaging they were the first to show that human MSCs migrate to the marginal zone of the spleen and regulate donor $\mathrm{T}$ cell proliferation preserving allo-specific graft-versus-leukemia response [63]. These results highlight the feasibility of tracking QDs-labeled
MSCs in vivo and open opportunities for mechanism of action investigations in other disease models.

Previously human MSCs were labeled with various QDs, including QDs525, QDs565, QDs585, QDs605, QDs655, and QDs800 [64, 65]. In our study we for the first time showed that QDs label human bone marrow MSC body, cell cytoplasmic protrusions and secreted ECM, thus revealing a more detailed MSC culture picture. QD size and charge are the key factors influencing QDs loading [66]. All this, including MSC tissue of origin, could explain the exclusive localization of QDs both inside and outside human bone marrow MSCs in vitro. Additionally, we for the first time used FLIM method to observe QDs localization in/on the extracellular matrix and showed that by analyzing mean fluorescence lifetime of QDs it is possible to identify and distinguish localization of QDs in various extracellular and intracellular structures without additional staining assays.

Recently we have demonstrated that QDs behave similarly in skin MSCs [25] indicating the potential universality of MSC staining method we are applying. However, several authors concluded that the characteristics of MSCs are tissue source-dependent. Al-Nbaheen et al. showed that human skin MSCs and human bone marrow MSCs exhibit differences in their proliferation, differentiation and molecular phenotype, which should be taken into consideration when planning their use in clinical protocols [67]. Liu et al. showed that human bone marrow MSCs and human skin MSCs differ in growth characteristics, gene expression and cytokine secretion profiles [68]. Reinisch et al. showed that human bone marrow, but not skin MSCs spontaneously formed hematopoietic niche in vivo [69]. Therefore, it might be too early to claim that all MSCs irrespective of origin would behave the same and the more precise experiments are required additionally for sufficient and conclusive evaluation of results.

\section{Conclusions}

In this study we successfully labeled human bone marrow MSCs with QDs without delivering vehicles. Human bone marrow MSC culture density is one of the key factors determining QDs localization: in more dense culture QDs preferably label MSC extraordinary filopodia-like and extracellular structures. To our best knowledge, we for the first time labeled MSC extracellular matrix with non-targeted carboxylated quantum dots and showed FLIM advantages for distinguishing QDs, allowing calculation of their mean photoluminescence lifetime and identifying their localization in/on intracellular and extracellular structures without additional staining techniques. These results are promising in fundamental stem cell biology as well as cellular therapy, anticancer drug 
delivery and provide a basis for further tissue engineering investigations.

\section{Methods Study design}

This study was performed in parallel with the study of mesenchymal stem cell (MSC) genetic characteristics published previously [31]. MSCs were isolated from bone marrow of 3 healthy donors. All donors have signed an Informed patient consent approved by the Vilnius Regional Committee of Biomedical Research (Lithuania, 2011-09-06 permission No. 158200-09-381-104). Isolated MSCs were expanded in vitro, characterized as proposed by The International Society for Cellular Therapy [31] and labeled with quantum dots (QDs). In this study, the QDs uptake dynamics, cytotoxity and biodistribution at subcellular level were investigated. The experiments were performed in the way to meet all three criteria for genuine replication [70]: (1) different donor cells randomized to flasks/wells independently, (2) different donor cells treated independently and no spillover, (3) different donor cells cultivated independently, thus, were not influencing each other.

\section{MSCs extraction using red blood cell lysis}

MSCs were isolated from $6 \mathrm{ml}$ (1 vacutainer) of bone marrow aspirate using red blood cell lysis method as previously described [34]. Briefly, all volume of vacutainer (6 ml) was transferred to the $50 \mathrm{ml}$ conical centrifugation tube (BD Biosciences, France) and Erythrocyte lysis buffer (Qiagen $\mathrm{GmbH}$, Germany) was added in the proportion 1:5. The tube was mixed for $1 \mathrm{~min}$ and centrifuged at $480 \times g$ for $5 \mathrm{~min}$. After centrifugation the top layer was discarded and the pellet was resuspended with $5 \mathrm{~mL}$ of RPMI 1640 medium (Gibco, Great Britain) and washed twice using centrifugation. All cells were seeded into $75 \mathrm{~cm}^{2}$ ventilated flask and cultivated for $24 \mathrm{~h}$ in the Dulbecco's Modified Eagle Medium (Lonza, Belgium) containing $10 \%$ of fetal bovine serum (FBS) (Invitrogen, USA) at $37{ }^{\circ} \mathrm{C}$ under a humidified $5 \% \mathrm{CO}_{2}$ atmosphere allowing the cells to adhere to the culture flask.

\section{MSCs cultivation}

Non-adherent cells were removed after $24 \mathrm{~h}$ by washing with phosphate buffered saline (PBS) solution (Gibco, USA). Human MSC basal medium (StemCell Technologies Inc., Canada) containing $10 \%$ of FBS for human MSCs (StemCell technologies Inc., Canada) was used for subsequent cultivation of MSCs. The medium was changed every 3-4 days. When adherent cells became subconfluent, MSCs were treated with trypsin-EDTA
(Gibco, USA), washed twice with PBS, calculated and seeded in the new $75 \mathrm{~cm}^{2}$ (BD Biosciences, France) flasks under the density of 4000 cells per $\mathrm{cm}^{2}$. The cells were incubated in a humidified $5 \% \mathrm{CO}_{2}$ incubator at $37{ }^{\circ} \mathrm{C}$. All procedures were performed in the class II vertical laminar safety cabinet (Kojair, Singapore). MSCs from all donors were subcultured and investigated at passage 3 .

\section{MSCs staining with Oil Red 0}

Samples were stained with $0.5 \%$ Oil Red O stain dissolved in isopropanol. Before the procedure Oil Red O solution was mixed with PBS in proportions 3:2 and then filtered with a sterile polyvinylidene Rotilabo $^{\circledR}$-syringe filters (Carl Roth $\mathrm{GmbH}+\mathrm{Co}$. KG, Germany) with $0.22 \mu \mathrm{m}$ pore size.

\section{Labeling MSCs with quantum dots}

MSCs were labeled using $\operatorname{Qdot}^{\circledR} 625$ ITK $^{\mathrm{TM}}$ Carboxyl quantum dots (QDs) with a photoluminescence (PL) peak at $625 \mathrm{~nm}$ (Invitrogen, USA). They are amphiphilic polymer coated CdSe/ZnS QDs with carboxyl groups, average hydrodynamic diameter of $14.2 \mathrm{~nm}$ and zeta potential $-32.97 \mathrm{mV}$. A layer covering QDs allows facile dispersion of the quantum dots in aqueous solutions with retention of their optical properties [71]. For more physicochemical characteristics of QDs, view supplementary information (Additional file 5). To evaluate QDs uptake dynamics, intracellular and extracellular localization, MSCs were harvested at P2 and seeded at a density of 5000 cells $/ \mathrm{cm}^{2}$ and 20,000 cells $/ \mathrm{cm}^{2}$ (for extracellular localization evaluation) in 8-well chambered cover-slips (Nunc, USA) for confocal fluorescence microscopy and allowed to grow for 1 day. Then MSCs were incubated in full serum media with QDs $(8 \mathrm{nM})$ over a time course ranging from $15 \mathrm{~min}$ to $24 \mathrm{~h}\left(37^{\circ} \mathrm{C}, 5 \% \mathrm{CO}_{2}\right)$.

\section{Analysis of QDs uptake and viability of QDs-labeled MSCs}

For quantitative analysis of QDs uptake, MSCs were seeded at a density of 20,000 cells $/ \mathrm{cm}^{2}$ in 12 -well plates (TPP, Switzerland) and allowed to grow for 2-3 days. Then MSCs were incubated with QDs $(8 \mathrm{nM})$ over a time course ranging from 1 to $24 \mathrm{~h}\left(37^{\circ} \mathrm{C}, 5 \% \mathrm{CO}_{2}\right)$. Flow cytometric analysis was carried out with a FACSort (BD Biosciences, USA). The data were analyzed with FlowJo (Tree Star, Ashland, OR) software. A minimum of 10000 viable cells were measured per sample. Using forward and side scatter profiles and propidium iodide staining, debris and dead cells were gated out, respectively. Viability was calculated as a percentage of viable cells per sample. The results were presented as mean \pm SD from three independent experiments. 
Imaging of QDs distribution in MSC culture

After indicated time of incubation, cells were routinely rinsed 3 times with pre-warmed human MSC basal medium (StemCell Technologies Inc., Canada) containing 10\% of FBS for human MSCs (StemCell technologies Inc., Canada) and then were analyzed using a confocal laser scanning microscope (Nikon Eclipse TE2000-S, C1 plus, Nikon, Tokyo, Japan) equipped with $\mathrm{CO}_{2}$ Microscope Stage Incubation System (OkoLab, Italy). Additionally, DIC and phase contrast microscopy were used to visualize the morphological characteristics of MSC treated with QDs. A diode laser for $405 \mathrm{~nm}$ and an argon laser for $488 \mathrm{~nm}$ excitation coupled with a $60 \times$ NA 1.4 oil immersion objective (Plan Apo VC, Nikon, Japan) were used for all measurements. To detect Hoechst (Sigma Aldrich, USA) fluorescence emission $\left(\lambda_{\text {ex }}=405 \mathrm{~nm}\right)$ the $450 / 35 \mathrm{~nm}$ band pass filter was used. Fluorescence of Alexa-Fluor $488 \mathrm{~nm}$-conjugated transferrin (Invitrogen, USA), Alexa-Fluor $488 \mathrm{~nm}$-conjugated phalloidin Invitrogen, USA) was detected using a 515/30 band pass filter $\left(\lambda_{\mathrm{ex}}=488 \mathrm{~nm}\right)$ as well as fluorescence of mouse antihuman CD44 antibody conjugated with Alexa-Fluor 488 (Thermo Fisher Scientific, USA) and fibronectin (Gibco, Thermo Fisher Scientific, USA). To visualize QDs fluorescence $\left(\lambda_{\mathrm{ex}}=488 \mathrm{~nm}\right)$ the $605 / 75 \mathrm{~nm}$ band pass filter was applied. Laser scanning was controlled by the Nikon EZ-C1 software; individual color channels were recorded separately to minimize spectrum overlap. The images were further processed using the EZ-C1 Bronze version 3.80 (Nikon, Japan) and ImageJ 1.41 software (NIH, USA).

\section{Fluorescence-lifetime imaging microscopy (FLIM) analysis}

FLIM images were obtained using Lifetime and FCS Upgrade for Nikon C1si (PicoQuant GmbH, Berlin, Germany). The imaging system was composed of a pulsed diode laser $(405 \mathrm{~nm})$ with a pulse width of $39 \mathrm{ps}$ and a repetition rate of $10 \mathrm{MHz}$. Detected photons were counted by a time correlated single-photon counter PicoHarp 300 (PicoQuant GmbH, Berlin, Germany). Excited states' PL lifetime signal of QDs in MSCs was investigated with a single channel unit of single photon-counting avalanche photodiodes (SPAD) at a spectral range of $650 \pm 75 \mathrm{~nm}$. Each one of FLIM images was acquired by collecting 1000 counts at the peak value. The image resolution was fixed at $512 \times 512$ pixels and images reconstruction work was performed using a three-exponential fitting model (SymPhoTime software, PicoQuant $\mathrm{GmbH}$, Germany).

\section{Additional files}

Additional file 1. Movie showing living mesenchymal stem cells under effect of trypsin after incubation with QDs for $24 \mathrm{~h}$.

Additional file 2. Living mesenchymal stem cells movie showing staining of extracellular matrix and intracellular accumulation of QDs. ROI 1 and 2 marking areas with stained ECM.

Additional file 3. 3D (z-stack) reconstruction movie showing MSCs after incubation with QDs for $6 \mathrm{~h}$.

Additional file 4. 3D (z-stack) reconstruction movie showing MSCs after incubation with QDs for $24 \mathrm{~h}$.

Additional file 5. Characterization of carboxylated QDs. (a) Image of QDs was made by Philips CM200 Field emission transmission electron microscope (TEM) equipped with light element EDX detector and Gatan Imaging Filter for PEELS and Energy Filtered TEM. Scale bar $20 \mathrm{~nm}$. (b) 3D topography image of QDs was registered on mica surface with Innova atomic force microscope in the tapping mode using silicon nitride probes MPP12283. (c) Hydrodynamic size distribution of QDs was measured using a dynamic light scattering device Zeta Plus PALS. (d) The steady state absorption and photoluminescence spectra were recorded $\left(\lambda_{\text {ex }}=625 \mathrm{~nm}\right)$ on Cary 50 UV-Vis spectrophotometer and Cary Eclipse fluorimeter, respectively. (e) PL decay curve of QDs was registered using FLS920 spectrometer equipped with $405 \mathrm{~nm}$ (66.9 ps) pulsed laser.

\section{Abbreviations}

DIC: differential interference contrast; ECM: extracellular matrix; FBS: fetal bovine serum; FLIM: fluorescence-lifetime imaging microscopy; MRI: magnetic resonance imaging; MSCs: mesenchymal stem cells; PBS: phosphate buffered saline; PL: photoluminescence; QDs: quantum dots; ROI: region of interest; SD: standard deviation; SPIO: superparamagnetic iron oxide; TEM: transmission electron microscope.

\section{Authors' contributions}

GK isolated and cultivated cells for experiments, analyzed the data, drafted and wrote the manuscript. KV performed imaging of QDs distribution in cell culture, analyzed the data, revised the manuscript. PM characterized quantum dots by atomic force microscopy, dynamic light scattering, steady state and time-resolved fluorescence spectroscopy, performed cell labeling with QDs, analyzed data, prepared images and revised manuscript. JM performed FLIM experiments, analyzed data, prepared images. SS performed QDs uptake dynamics and cell viability analysis. ZG isolated cells. RR designed the study, revised the manuscript. All authors read and approved the final manuscript.

\section{Author details}

${ }^{1}$ Biobank, National Cancer Institute, Baublio Str. 3b, 08406 Vilnius, Lithuania. ${ }^{2}$ Biomedical Physics Laboratory, National Cancer Institute, Baublio Str. 3b, 08406 Vilnius, Lithuania. ${ }^{3}$ Department of Chemistry and Bioengineering, Vilnius Gediminas Technical University, Sauletekis Ave. 11, 10223 Vilnius, Lithuania. ${ }^{4}$ Laboratory of Immunology, National Cancer Institute, Baublio Str. 3b, 08406 Vilnius, Lithuania. ${ }^{5}$ Biophotonics Group of Laser Research Center, Faculty of Physics, Vilnius University, Sauletekis Ave. 9, 10222 Vilnius, Lithuania.

\section{Acknowledgements}

Vitalijus Karabanovas is grateful for dedicated funding from the project "Postdoctoral Fellowship Implementation in Lithuania" (VP1-3.1-SMM-01-V-01-001). The authors would like to thank Dr. Ruta Araminaite for carrying out TEM measurements.

\section{Competing interests}

The authors declare that they have no competing interests. 


\section{Availability of data and materials}

All data generated or analysed during this study are included in this published article and its additional files.

\section{Consent for publication}

All authors agree to publication.

\section{Ethics approval and consent to participate}

All donors included in this study have signed an informed patient consent approved by the Vilnius Regional Committee of Biomedical Research (Lithuania, 2011-09-06 permission No. 158200-09-381-104).

\section{Funding}

This research was partially supported by the Norwegian Financial Mechanism and the Republic of Lithuania within the project "Multifunctional nanoparticles for specific non-invasive early diagnostics and treatment of cancer" (Contract No. LT0036) and by the European Union Structural Funds \& the Ministry of Education and Science of the Republic of Lithuania Grant "Programming cells and management of tumor microenvironment for personal therapy in oncology-LASTER" (No. VP1-3.1-SMM-10-V-02-027).

\section{Publisher's Note}

Springer Nature remains neutral with regard to jurisdictional claims in published maps and institutional affiliations.

\section{Received: 11 September 2018 Accepted: 26 February 2019} Published online: 13 March 2019

\section{References}

1. Nombela-Arrieta C, Ritz J, Silberstein LE. The elusive nature and function of mesenchymal stem cells. Nat Rev Mol Cell Biol. 2011;12:126-31.

2. Zhao Q, Ren H, Han Z. Mesenchymal stem cells: immunomodulatory capability and clinical potential in immune diseases. J Cell Immunother. 2016:2:3-20.

3. Squillaro T, Peluso G, Galderisi U. Clinical trials with mesenchymal stem cells: an update. Cell Transplant. 2016;25:829-48.

4. Shah K. Mesenchymal stem cells engineered for cancer therapy. Adv Drug Deliv Rev. 2012;64:739-48.

5. Mohammadi M, Jaafari MR, Mirzaei HR, Mirzaei H. Mesenchymal stem cell: a new horizon in cancer gene therapy. Cancer Gene Ther. 2016;23:285-6.

6. Hagenhoff A, Bruns CJ, Zhao Y, von Lüttichau I, Niess H, Spitzweg C, et al. Harnessing mesenchymal stem cell homing as an anticancer therapy. Expert Opin Biol Ther. 2016;16:1079-92.

7. Schmuck EG, Koch JM, Centanni JM, Hacker TA, Braun RK, Eldridge M, et al. Biodistribution and clearance of human mesenchymal stem cells by quantitative three-dimensional cryo-imaging after intravenous infusion in a rat lung injury model. Stem Cells Transl Med. 2016;5:1668-75.

8. Kim J, Chhour P, Hsu J, Litt HI, Ferrari VA, Popovtzer R, et al. Use of nanoparticle contrast agents for cell tracking with computed tomography. Bioconjugate Chem. 2017;28:1581-97.

9. Kim SM, Jeong CH, Woo JS, Ryu CH, Lee J-H, Jeun S-S. In vivo nearinfrared imaging for the tracking of systemically delivered mesenchyma stem cells: tropism for brain tumors and biodistribution. Int J Nanomed. 2015;11:13-23.

10. Naseroleslami M, Parivar K, Khoei S, Aboutaleb N. Magnetic resonance imaging of human-derived amniotic membrane stem cells using PEGylated superparamagnetic iron oxide nanoparticles. Cell J. 2016;18:332-9.

11. Scharf A, Holmes S, Thoresen M, Mumaw J, Stumpf A, Peroni J. Superparamagnetic iron oxide nanoparticles as a means to track mesenchymal stem cells in a large animal model of tendon injury. Contrast Media Mol Imaging. 2015;10:388-97.

12. Nucci LP, Silva HR, Giampaoli V, Mamani JB, Nucci MP, Gamarra LF. Stem cells labeled with superparamagnetic iron oxide nanoparticles in a preclinical model of cerebral ischemia: a systematic review with metaanalysis. Stem Cell Res Ther. 2015;6:27.
13. Santoso MR, Yang PC. Magnetic nanoparticles for targeting and imaging of stem cells in myocardial infarction. Stem Cells Int. 2016;1:1. https://doi. org/10.1155/2016/4198790.

14. Ma Y, Ji Y, You M, Wang S, Dong Y, Jin G, et al. Labeling and long-term tracking of bone marrow mesenchymal stem cells in vitro using NaYF4:Yb3 +, Er3 + upconversion nanoparticles. Acta Biomater. 2016:42:199-208.

15. Duan X, Wang Y, Zhang F, Lu L, Cao M, Lin B, et al. Superparamagnetic iron oxide-loaded cationic polymersomes for cellular mr imaging of therapeutic stem cells in stroke. J Biomed Nanotechnol. 2016;12:2112-24.

16. Zhou B, Li D, Qian J, Li Z, Pang P, Shan H. MR tracking of SPIO-labeled mesenchymal stem cells in rats with liver fibrosis could not monitor the cells accurately. Contrast Media Mol Imaging. 2015;10:473-80.

17. Liu S, Tay LM, Anggara R, Chuah YJ, Kang Y. Long-term tracking mesenchymal stem cell differentiation with photostable fluorescent nanoparticles. ACS Appl Mater Interfaces. 2016:8:11925-33.

18. Collins MC, Gunst PR, Cascio WE, Kypson AP, Muller-Borer BJ. Labeling and imaging mesenchymal stem cells with quantum dots. In: Soloviev M, editor. Nanoparticles in biology and medicine: methods and protocols. Totowa: Humana Press; 2012. p. 199-210.

19. Gao X, Yang L, Petros JA, Marshall FF, Simons JW, Nie S. In vivo molecular and cellular imaging with quantum dots. Curr Opin Biotechnol. 2005;16:63-72.

20. Hsieh S-C, Wang F-F, Lin C-S, Chen Y-J, Hung S-C, Wang Y-J. The inhibition of osteogenesis with human bone marrow mesenchymal stem cells by CdSe/ZnS quantum dot labels. Biomaterials. 2006;27:1656-64.

21. Shah BS, Mao JJ. Labeling of mesenchymal stem cells with bioconjugated quantum dots. In: Shah K, editor. Molecular imaging: methods and protocols. Totowa: Humana Press; 2011. p. 61-75.

22. Wu Y, Zhou B, Xu F, Wang X, Liu G, Zheng $L$, et al. Functional quantum dot-siRNA nanoplexes to regulate chondrogenic differentiation of mesenchymal stem cells. Acta Biomater. 2016;46:165-76.

23. Li J, Lee WY, Wu T, Xu J, Zhang K, Li G, et al. Multifunctional quantum dot nanoparticles for effective differentiation and long-term tracking of human mesenchymal stem cells in vitro and in vivo. Adv Healthcare Mater. 2016:5:1049-57.

24. Saulite L, Dapkute D, Pleiko K, Popena I, Steponkiene S, Rotomskis R, et al. Nano-engineered skin mesenchymal stem cells: potential vehicles for tumour-targeted quantum-dot delivery. Beilstein J Nanotechnol. 2017;8:1218-30.

25. Dapkute D, Steponkiene S, Bulotiene D, Saulite L, Riekstina U, Rotomskis R. Skin-derived mesenchymal stem cells as quantum dot vehicles to tumors. Int J Nanomed. 2017;12:8129-42.

26. Saulite L, Pleiko K, Popena I, Dapkute D, Rotomskis R, Riekstina U. Nanoparticle delivery to metastatic breast cancer cells by nanoengineered mesenchymal stem cells. Beilstein J Nanotechnol. 2018;9:321-32.

27. Ohyabu Y, Kaul Z, Yoshioka T, Inoue K, Sakai S, Mishima H, et al. Stable and nondisruptive in vitro/in vivo labeling of mesenchymal stem cells by internalizing quantum dots. Hum Gene Ther. 2009;20:217-24.

28. Tao Z-W, Favreau JT, Guyette JP, Hansen KJ, Lessard J, Burford E, et al. Delivering stem cells to the healthy heart on biological sutures: effects on regional mechanical function. J Tissue Eng Regen Med. 2017;11:220-30.

29. Hass R, Kasper C, Böhm S, Jacobs R. Different populations and sources of human mesenchymal stem cells (MSC): a comparison of adult and neonatal tissue-derived MSC. Cell Commun Signal. 2011;9:12.

30. Kim HJ, Park J-S. Usage of Human Mesenchymal Stem Cells in Cell-based Therapy: advantages and Disadvantages. Dev Reprod. 2017;21:1-10.

31. Kundrotas G, Gasperskaja E, Slapsyte G, Gudleviciene Z, Krasko J, Stumbryte A, et al. Identity, proliferation capacity, genomic stability and novel senescence markers of mesenchymal stem cells isolated from low volume of human bone marrow. Oncotarget. 2016;7:10788-802.

32. Karabanovas V, Zitkus Z, Kuciauskas D, Rotomskis R, Valius M. Surface properties of quantum dots define their cellular endocytic routes, mitogenic stimulation and suppression of cell migration. J Biomed Nanotechnol. 2014;10:775-86.

33. Damalakiene L, Karabanovas V, Bagdonas S, Rotomskis R. Fluorescencelifetime imaging microscopy for visualization of quantum dots' endocytic pathway. Int J Mol Sci. 2016;17:473.

34. Gudleviciene Z, Kundrotas G, Liudkeviciene R, Rascon J, Jurga M. Quick and effective method of bone marrow mesenchymal stem cell extraction. Open Med (Wars). 2014;10:44-9. 
35. Dominici M, Le Blanc K, Mueller I, Slaper-Cortenbach I, Marini FC, Krause DS, et al. Minimal criteria for defining multipotent mesenchymal stromal cells. The International Society for Cellular Therapy position statement. Cytotherapy. 2006;8:315-7.

36. Sabapathy $\vee$, Mentam J, Jacob PM, Kumar S. Noninvasive optical imaging and in vivo cell tracking of indocyanine green labeled human stem cells transplanted at superficial or in-depth tissue of SCID mice. Stem Cells Int. 2015. https://doi.org/10.1155/2015/606415.

37. Winnik FM, Maysinger D. Quantum dot cytotoxicity and ways to reduce it. Acc Chem Res. 2013;46:672-80.

38. Wang G, Zeng G, Wang C, Wang H, Yang B, Guan F, et al. Biocompatibility of quantum dots (CdSe/ZnS) in human amniotic membrane-derived mesenchymal stem cells in vitro. Biomed Papers. 2015;159:227-33.

39. Muller-Borer BJ, Collins MC, Gunst PR, Cascio WE, Kypson AP. Quantum dot labeling of mesenchymal stem cells. J Nanobiotechnol. 2007;5:9.

40. Damalakiene L, Karabanovas V, Bagdonas S, Valius M, Rotomskis R. Intracellular distribution of nontargeted quantum dots after natural uptake and microinjection. Int J Nanomed. 2013;8:555-68.

41. Okolicsanyi RK, Camilleri ET, Oikari LE, Yu C, Cool SM, van Wijnen AJ, et al. Human mesenchymal stem cells retain multilineage differentiation capacity including neural marker expression after extended in vitro expansion. PLoS ONE. 2015;10:e0137255.

42. Seleverstov O, Zabirnyk O, Zscharnack M, Bulavina L, Nowicki M, Heinrich J-M, et al. Quantum dots for human mesenchymal stem cells labeling. A size-dependent autophagy activation. Nano Lett. 2006;6:2826-32.

43. Lei Y, Tang H, Yao L, Yu R, Feng M, Zou B. Applications of mesenchymal stem cells labeled with tat peptide conjugated quantum dots to cell tracking in mouse body. Bioconjugate Chem. 2008;19:421-7.

44. Fink T, Zachar V. Adipogenic differentiation of human mesenchymal stem cells. In: Vemuri M, Chase LG, Rao MS, editors. Mesenchymal stem cell assays and applications. Totowa: Humana Press; 2011. p. 243-51.

45. Heid H, Rickelt S, Zimbelmann R, Winter S, Schumacher H, Dörflinger Y. Lipid droplets, perilipins and cytokeratins - unravelled liaisons in epithelium-derived cells. PLoS ONE. 2013;8:e63061.

46. Bajek A, Olkowska J, Walentowicz-Sadłecka M, Walentowicz P, Sadłecki P, Grabiec M, et al. High quality independent from a donor: human amniotic fluid derived stem cells - a practical analysis based on 165 clinical cases. J Cell Biochem. 2018;118:116-26.

47. He W, Elkhooly TA, Liu X, Cavallaro A, Taheri S, Vasilev K, et al. Silver nanoparticle based coatings enhance adipogenesis compared to osteogenesis in human mesenchymal stem cells through oxidative stress. J Mater Chem B. 2016:4:1466-79.

48. Jichuan Qiu, Deshuai Li, Xiaoning Mou, Jianhua Li, Weibo Guo, Shu Wang, et al. Effects of graphene quantum dots on the self-renewal and differentiation of mesenchymal stem cells. Adv Healthcare Mater. 2016;5:702-10.

49. Tencerova M, Kassem M. The bone marrow-derived stromal cells: commitment and regulation of adipogenesis. Front Endocrinol (Lausanne). 2016;7:127.

50. Wang K, Seo BR, Fischbach C, Gourdon D. Fibronectin mechanobiology regulates tumorigenesis. Cell Mol Bioeng. 2016;9:1-11.

51. Ferrari LF, Araldi D, Bogen O, Levine JD. Extracellular matrix hyaluronan signals via its CD44 receptor in the increased responsiveness to mechanical stimulation. Neuroscience. 2016;324:390-8.

52. Chen J, Pei Y, Chen Z, Cai J. Quantum dot labeling based on near-field optical imaging of CD44 molecules. Micron. 2010;41:198-202.

53. Ke C, Chen J, Guo Y, Chen ZW, Cai J. Migration mechanism of mesenchymal stem cells studied by QD/NSOM. Biochimica et Biophysica Acta (BBA) Biomembranes. 2015;1848:859-68.

54. Chen XD. Extracellular matrix provides an optimal niche for the maintenance and propagation of mesenchymal stem cells. Birth Defects Res C Embryo Today. 2010;90:45-54.
55. Köllmer M, KeskarV, HaukTG, Collins JM, Russell B, Gemeinhart RA. Stem cell-derived extracellular matrix enables survival and multilineage differentiation within superporous hydrogels. Biomacromol. 2012;13:963-73.

56. Harvey A, Yen T-Y, Aizman I, Tate C, Case C. Proteomic analysis of the extracellular matrix produced by mesenchymal stromal cells: implications for cell therapy mechanism. PLoS ONE. 2013;8:e79283.

57. Teti G, Cavallo C, Grigolo B, Giannini S, Facchini A, Mazzotti A, et al. Ultrastructural analysis of human bone marrow mesenchymal stem cells during in vitro osteogenesis and chondrogenesis. Microscopy Res Techn. 2012;75:596-604.

58. Dudakovic AD, Camilleri E, Riester SM, Lewallen EA, Kvasha S, Chen X, et al. High-resolution molecular validation of self-renewal and spontaneous differentiation in adipose-tissue derived human mesenchymal stem cells cultured in human platelet lysate. J Cell Biochem. 2014;115:1816-28.

59. Ishikawa-Ankerhold HC, Ankerhold R, Drummen GPC. Advanced fluorescence microscopy techniques -FRAP, FLIP, FLAP, FRET and FLIM. Molecules. 2012;17:4047-132.

60. Zhang L, Lei J, Liu J, Ma F, Ju H. Persistent luminescence nanoprobe for biosensing and lifetime imaging of cell apoptosis via time-resolved fluorescence resonance energy transfer. Biomaterials. 2015;67:323-34.

61. McCorry MC, Bonassar LJ. Fiber development and matrix production in tissue engineered menisci using bovine mesenchymal stem cells and fibrochondrocytes. Connect Tissue Res. 2017;58:329-41.

62. Han S, Li YY, Chan BP. Protease inhibitors enhance extracellular collagen fibril deposition in human mesenchymal stem cells. Stem Cell Res Ther. 2015;6:197.

63. Auletta JJ, Eid SK, Wuttisarnwattana P, Silva I, Metheny L, Keller MD, et al. Human mesenchymal stromal cells attenuate graft-versus-host disease and maintain graft-versus-leukemia activity following experimental allogeneic bone marrow transplantation. Stem Cells. 2015;33:601-14.

64. Ranjbarvaziri S, Kiani S, Akhlaghi A, Vosough A, Baharvand H, Aghdami N. Quantum dot labeling using positive charged peptides in human hematopoetic and mesenchymal stem cells. Biomaterials. 2011;32:5195-205.

65. Yang HN, Park JS, Jeon SY, Park W, Na K, Park K-H. The effect of quantum dot size and poly(ethylenimine) coating on the efficiency of gene delivery into human mesenchymal stem cells. Biomaterials. 2014;35:8439-49.

66. Rosen AB, Kelly DJ, Schuldt AJT, Lu J, Potapova IA, Doronin SV, et al. Finding fluorescent needles in the cardiac haystack: tracking human mesenchymal stem cells labeled with quantum dots for quantitative in vivo three-dimensional fluorescence analysis. STEM CELLS. 2007;25:2128-38.

67. Al-Nbaheen M, Vishnubalaji R, Ali D, Bouslimi A, Al-Jassir F, Megges M, Prigione A, Adjaye J, Kassem M, Aldahmash A. Human stromal (mesenchymal) stem cells from bone marrow, adipose tissue and skin exhibit differences in molecular phenotype and differentiation potential. Stem Cell Rev. 2013;9(1):32-43.

68. Liu R, Chang W, Wei H, Zhang K. Comparison of the biological characteristics of mesenchymal stem cells derived from bone marrow and skin. Stem Cells Int. 2016;2016:3658798.

69. Lazic SE, Clarke-Williams CJ, Munafò MR. What exactly is ' $N$ ' in cell culture and animal experiments? PLoS Biol. 2018;16(4):e2005282.

70. Reinisch A, Etchart N, Thomas D, Hofmann NA, Fruehwirth M, Sinha S, Chan CK, Senarath-Yapa K, Seo EY, Wearda T, Hartwig UF, Beham-Schmid C, Trajanoski S, Lin Q, Wagner W, Dullin C, Alves F, Andreeff M, Weissman IL, Longaker MT, Schallmoser K, Majeti R, Strunk D. Epigenetic and in vivo comparison of diverse MSC sources reveals an endochondral signature for human hematopoietic niche formation. Blood. 2015;125(2):249-60.

71. Rotomskis R, Jurgelènè Ž, Stankevičius M, Stankevičiūtè M, Kazlauskienè $\mathrm{N}$, Jokšas K, et al. Interaction of carboxylated CdSe/ZnS quantum dots with fish embryos: towards understanding of nanoparticles toxicity. Sci Total Environ. 2018;635:1280-91. 